\title{
Radiographic progression of hospital referred osteoarthritis of the hip
}

\author{
Joanna Ledingham, Stewart Dawson, Bryan Preston, George Milligan, Michael Doherty
}

\begin{abstract}
Various factors have been considered important in the rate of progression of osteoarthritis of the hip, though few data are available from large longitudinal studies.

One hundred and thirty six patients referred to hospital (85 women, 51 men; mean age 65 years, range $29-86$ years) with osteoarthritis of the hip (192 affected hips) were followed up for a median of 27 (range 3-72) months. One hundred and six patients (144 affected hips) were reviewed for a minimum of one year (median 28, range 12-72 months). Fifty three per cent of all osteoarthritic hips required an operation at a median of 14 (range 3-48) months from entry. All radiographs before and after entry were examined (median four films for each patient over a median of three years, range 0.5-19 years). Hips showing rapid radiographic progression on prospective films more often had superior migration, or an atrophic bone response; those with no progression more often had an indeterminate, medial, or axial migration pattern, protrusio or mild osteoarthritis at presentation. A higher occurrence of rapid progression was seen in women, in those of older age at symptom onset, and in hips with a higher Kellgren grade at entry; men more often showed no progression. Age at presentation, body mass index, symptom duration, or presence of chondrocalcinosis, hand osteoarthritis/ nodes, or Forestier's disease did not influence progression.
\end{abstract}

This study supports the contention that, at the time of hospital referral, certain patient characteristics and radiographic features at the hip may help to identify those at high risk of rapidly progressive hip osteoarthritis.

(Ann Rheum Dis 1993; 52: 263-267)

Debate continues with regard to the definition and classification of osteoarthritis. Hip osteoarthritis is usually characterised in terms of radiographic appearance, but differing emphasis has been placed on varying radiographic aspects and little is known of their prognostic significance. Subclassification has been according to the pattern of migration of the femoral head within the acetabulum, ${ }^{1-5}$ and the pattern of bone response to cartilage loss. The importance of these patterns with respect to outcome remains unclear, ${ }^{6-16}$ though medial migration is reportedly less 'severe' than superior migration, ${ }^{14}{ }^{17} 18$ and an atrophic bone response has been associated with poor outcome. ${ }^{5}$ The presence of chondrocalcinosis at other joint sites has also been associated with an atrophic bone response and poor outcome at the hip, ${ }^{19-21}$ and obesity has been incriminated in rapid progression. ${ }^{22}$

This study was therefore undertaken to evaluate the prognostic significance of the femoral head migration and bone response patterns. In addition, the influence of other factors such as obesity, presence of Heberden's nodes, polyarticular and pauciarticular interphalangeal osteoarthritis, osteoarthritis at other joint sites, Forestier's disease and chondrocalcinosis was assessed. The study was limited to patients with sufficiently severe problems to warrant referral to hospital.

\section{Patients and methods}

This study was approved by the local ethical committee and informed consent was obtained from all patients. Consecutive patients attending one rheumatology and three orthopaedic clinics (all general) with symptomatic hip osteoarthritis were recruited into the study over a two year period. Osteoarthritis of the hip was defined as the presence of radiographic change (joint space narrowing with osteophyte, with or without cyst formation, sclerosis, attrition) together with pain or clinical abnormalities, or both, at that site. Alternative arthropathies were excluded by clinical examination, blood tests, microscopy of synovial fluid, and radiographic screening. ${ }^{23}$ Each patient underwent a full rheumatological history and examination with particular emphasis on the presence of activity, rest, and nocturnal hip pain (each graded $0-3$ ), duration of symptoms, and past history of previous major trauma or operation on the hips. On examination, clinical abnormality consistent with osteoarthritis was noted for all non-axial joints. Nodal change was defined clinically as the presence of typical superolateral Heberden's or Bouchard's nodes, or both, affecting at least three rays of each hand. In each patient plain radiographs were taken of the pelvis (supine anteroposterior), hands (to include wrists), shoulders, knees (standing anteroposterior plus $20^{\circ}$ flexion lateral views), lumbar spine (anteroposterior and lateral), and thoracic spine 
(anteroposterior), together with any other clinically affected joint.

Initial films were examined and scored as previously described. ${ }^{23}$ The Kellgren and Lawrence grading system ${ }^{24}$ was used to assess radiographic severity of osteoarthritis at the hips and other joints: osteoarthritis was recorded as present in any joint scored as Kellgren grade 2 or more. Multiple large joint involvement was defined as at least one other large joint (for example, knee, shoulder) being affected in addition to the hip; polyarticular interphalangeal osteoarthritis was defined by radiographic involvement of interphalangeal joints in at least three rays of both hands. Chondrocalcinosis and Forestier's disease ${ }^{1}$ were recorded if present. The pattern of migration of the femoral head within the acetabulum was classified into superolateral, superomedial, superior intermediate, medial, axial, or indeterminate patterns. ${ }^{1}$ The bone response at each hip was categorised as either atrophic or hypertrophic ${ }^{5}$; an indeterminate category was used for hips showing a mixed pattern of bone response. In addition, individual features of osteoarthritis were graded 0-3 using a modification of Thomas's method $^{25}$ at specific sites. ${ }^{23}$

Each patient was reviewed at approximately six month intervals after recruitment when repeat rheumatological history and examination were undertaken. Patients gave a global assessment of change in their symptoms (better, same, worse), and pain scores at completion of the study were compared with

Table 1 Patient characteristics (total number of hips 192)

\begin{tabular}{ll}
\hline Characteristic & Value \\
\hline Median (range) age (years) & $65(29-85)$ \\
Median (range) duration of follow up & $24(3-72)$ \\
(months) & \\
Median (range) body mass index & $26 \cdot 54(18 \cdot 18-47 \cdot 77)$ \\
No (\%) with hip osteoarthritis & $61(45 \%)$ \\
Unilateral & $71(52 \%)$ \\
Bilateral & $4(3 \%)$ \\
Previous total hip replacement & $36(26 \%)$ \\
No (\%) with isolated osteoarthritis of & \\
the hip & $47(35 \%)$ \\
& \\
No (\%) with no radiographic & \\
osteoarthritis of the hand & \\
No (\%) with radiographic osteoarthritis & \\
at other sites & $17(13 \%)$ \\
Polyarticular interphalangeal & $71(51 \%)$ \\
Any interphalangeal & $42(30 \%)$ \\
First carpometacarpal joint & $42(30 \%)$ \\
Knee & $16(12 \%)$ \\
Glenohumeral & $14(10 \%)$ \\
No (\%) with nodal changes & $13(10 \%)$ \\
No (\%) with chondrocalcinosis & $9(6 \%)$ \\
No (\%) with Forestier's disease & \\
\hline
\end{tabular}

Table 2 Migration and bone response patterns for all hips followed prospectively and the 144 hips followed up for at least one year

\begin{tabular}{lcc}
\hline & $\begin{array}{l}\text { No (\%) of osteoarthritic hips } \\
(n=192)\end{array}$ & $\begin{array}{l}\text { No (\%) of osteoarthritic hips } \\
\text { after at least one year } \\
\text { follow up (n=144) }\end{array}$ \\
\hline Migration & \\
Superolateral & $79(41)$ & $58(40)$ \\
Superior intermediate & $51(27)$ & $38(26)$ \\
Superomedial & $20(10)$ & $14(10)$ \\
Medial & $16(8)$ & $12(8)$ \\
Axial & $3(2)$ & $2(1)$ \\
Indeterminate & $23(12)$ & $20(14)$ \\
Bone response & $35(18)$ & $21(15)$ \\
Atrophic & $31(16)$ & $22(15)$ \\
Hypertrophic & $126(66)$ & $101(70)$ \\
Intermediate & & \\
\hline
\end{tabular}

the initial scores. Functional status was assessed at each visit using the Steinbrocker index and reported exercise tolerance, and radiographs of both hips (and any new symptomatic, clinically affected joint site) were obtained. Radiographs taken immediately before an operation (where available) were assessed in those patients undergoing hip replacement within six months of study entry.

Individual radiographic features at the hip (joint space narrowing, osteophytosis, cyst formation, sclerosis, and attrition) were assessed for change (increased, same, decreased) at each six month interval and a global assessment of change (no change, slow, stepwise, or rapid progression) was made at the end of the study. In addition, radiographs taken before entry to the study were assessed by the same method. The last available radiograph for each patient, at completion of the study, was assessed in an identical fashion to the study entry film and changes in scores for each individual feature obtained. Reproducibility for this scoring method (for the same assessors) has already been shown to be good for attrition and narrowing and moderate for osteophyte, sclerosis, and cysts. ${ }^{23}$

Statistical analysis was performed using the $\chi^{2}$ (with Yates's correction) and Fisher's tests as appropriate. Odds ratios were calculated with respect to the presence or absence of putative risk factors.

\section{Results}

\section{PATIENT CHARACTERISTICS}

One hundred and thirty six patients (85 women, 51 men; mean age 65 years, range 29-86 years) were recruited into the study (table 1) and follow up radiographs were obtained of at least one hip. In a further 30 patients ( 21 women, nine men) only retrospective radiographic data were available. At study entry $45 \%$ had unilateral and $52 \%$ bilateral hip osteoarthritis; a further four patients were affected in their second hip after a previous hip replacement. A total of 192 osteoarthritic hips were prospectively reviewed, 144 for a minimum of 12 months. Fifty seven per cent of hips had severe, $28 \%$ moderate, and $15 \%$ mild radiographic changes (corresponding to Kellgren grades 4,3 , and 2 respectively) at entry and the occurrence of the various migration and bone response patterns are shown in table 2 . Thirty five (18\%) hips showing definite radiographic osteoarthritis were asymptomatic; these included hips with marked attrition and similar severity of change as in the contralateral symptomatic hip.

Only $15 \%$ of the osteoarthritic hips showed radiographic change as assessed by a change in Kellgren grade; most of these were Kellgren grade 3 (moderate osteoarthritis) at entry. When individual features were assessed, changes were seen in hips with mild, moderate, and severe changes at entry. Sixty four per cent of hips showed a change in at least one radiographic feature and using the method of global assessment of change, progression was seen in $47 \%$. 
Table 3 Odds ratios for rapid, slow, and no radiographic progression for putative risk factors

\begin{tabular}{llclr}
\hline Progression & Risk factor & $\begin{array}{l}\text { Odds } \\
\text { ratio }\end{array}$ & $\begin{array}{l}95 \% \text { Confidence } \\
\text { interval }\end{array}$ & $\begin{array}{c}p \\
\text { Value }\end{array}$ \\
\hline Rapid & Female sex & 2.53 & 0.91 to 7.41 & 0.05 \\
& Atrophic bone response & 8.31 & 3.18 to 21.98 & $<0.001$ \\
& Superior migration & 9.00 & 1.24 to 183.52 & 0.011 \\
Slow & Moderate osteoarthritis & 2.88 & 1.27 to 6.52 & 0.005 \\
& Intermediate bone response & 2.52 & 0.97 to 6.77 & 0.037 \\
& Male sex & 2.33 & 1.2 to 4.34 & 0.006 \\
& Mild osteoarthritis & 15.05 & 3.31 to 94.99 & $<0.001$ \\
& Medial/axial migration & 3.71 & 1.09 to 13.83 & 0.018 \\
& Indeterminate migration & 11.41 & 2.47 to 72.79 & $<0.001$ \\
& Superior migration & 0.13 & 0.05 to 0.35 & $<0.001$ \\
\hline
\end{tabular}

Table 4 Factors associating with an operation for hip replacement

\begin{tabular}{lrlr}
\hline Factor & $\begin{array}{l}\text { Odds } \\
\text { ratio }\end{array}$ & $\begin{array}{l}\text { 95\% Confidence } \\
\text { intervals }\end{array}$ & p Value \\
\hline Symptoms & & & \\
$\quad$ Rest pain & 2.80 & 1.49 to 5.31 & $<0.001$ \\
$\quad \begin{array}{l}\text { Night pain } \\
\quad \text { Patient assessment of change (worse versus better or }\end{array}$ & 11.73 & 1.45 to 5.16 & $<0.001$ \\
$\quad$ no change & 2.68 & 1.29 to 24.73 & $<0.001$ \\
$\begin{array}{l}\text { Decrease in exercise tolerance } \\
\text { Atrophic bone response }\end{array}$ & 3.13 & 1.28 to 7.69 & 0.004 \\
Radiographic change & & & 0.006 \\
$\quad$ Any (rapid, stepwise, slow) & 2.19 & 1.18 to 4.08 & 0.008 \\
$\quad$ Rapid & 5.83 & 1.90 to 19.11 & $<0.001$ \\
\hline
\end{tabular}

GLOBAL RADIOGRAPHIC CHANGE

Owing to the small numbers of hips showing medial or axial patterns of migration, these two groups were merged for statistical analysis. Table 3 gives the odds ratios for significant risk factors. Hips showing rapid radiographic progression more commonly had severe changes at study entry (Kellgren grade 4), superior migration patterns, or atrophic bone response. Women and patients with an older age of symptom onset also more often showed rapid radiographic progression, whereas men more often showed no progression. Hips showing no progression more commonly had an indeterminate, medial, or axial migration pattern, protrusio deformity, or mild osteoarthritis (Kellgren grade 2) at study entry. Slow progression occurred more often in hips with an intermediate bone response pattern and moderate osteoarthritis (Kellgren grade 3 ) at entry. Body mass index, age at presentation, symptom duration, presence of nodal change, polyarticular or pauciarticular interphalangeal, multiple large joint, isolated hip osteoarthritis, Forestier's disease, and chondrocalcinosis did not influence radiographic progression.

\section{INDIVIDUAL RADIOGRAPHIC FEATURES}

Changes in all individual radiographic features occurred more often in hips with the superolateral migration pattern. Women more often showed changes in the Kellgren grade, narrowing, attrition and cysts. Hips with mild osteoarthritis (Kellgren grade 2) at entry, and the medial and axial migration patterns more commonly showed no change in any individual feature. As with the global assessment of change, other patient characteristics did not associate with change in radiographic features.

HIP REPLACEMENT

One hundred and two (53\%) of the 192 osteoarthritic hips underwent an operation for hip replacement at a median of 14 (range 3-48) months from entry. Of the 144 hips followed up for at least one year (median follow up 28, range $12-72$ months), $68(47 \%)$ had an operation at a median of 20 (range 12-48) months from entry.

Table 4 shows factors associating with hip replacement. An operation was more commonly undertaken in hips with an atrophic bone response and higher scores at entry for overall severity, narrowing, attrition, osteophyte, and cysts. Patients with rest and night pain, higher overall pain scores, and higher Steinbrocker grades at entry also commonly required an operation, as did those with increased pain scores and reduced reported exercise tolerance during the study period. Hips showing global radiographic change, and in particular those which developed rapid change, more commonly underwent an operation for hip replacement. Age, sex, body mass index, nodal change, osteoarthritis at other sites (present or absent), Forestier's disease, and chondrocalcinosis showed no associations with the need for a hip operation.

PAIN

Most patients ( $88 \%$ ) regarded their symptoms as worsening with time and $66 \%$ of patients had increased pain scores. Pain scores increased more commonly in women and in hips with superolateral migration, radiographic progression, and changes in Kellgren grade and attrition. Improved pain scores were seen more commonly in men, patients with chondrocalcinosis, and in hips with mild osteoarthritis at entry and showing no radiographic progression. Other patient characteristics did not associate with changes in pain scores or with the patient's assessment of change.

\section{DISABILITY}

Most patients $(90 \%)$ showed no change in function, assessed in terms of the Steinbrocker index, during the study period. Function improved in four patients and deteriorated in 13 (all women). Deterioration was more common in patients with worsening symptoms, increased pain scores, and radiographic progression; no increase in Steinbrocker score was seen in patients with no radiographic progression. Reported change in exercise tolerance was found to be a more sensitive measure of functional change, however, with $31 \%$ describing reduction and $62 \%$ no change in their exercise tolerance.

\section{Discussion}

This study was primarily designed to assess the influence of a variety of clinical and radiographic features, present at the time of hospital referral, on radiographic change in hip osteoarthritis. Estimates of associated disability were made using the Steinbrocker index and reported exercise tolerance, however. Although reported maximum walking distance must be 
interpreted with a certain degree of caution as many factors, including psychological factors, may influence patient reporting, this was found to be a more sensitive measure of change in disability than the Steinbrocker index and was found to associate with radiographic change and an operation for hip replacement.

This study was not designed to assess factors influencing decisions of individual orthopaedic surgeons to operate and hence data relating to hip replacement as an end point must also be interpreted with a certain degree of caution. As would perhaps be expected, however, factors associated with a hip operation were primarily severe and increasing pain, disability, and radiographic change (in particular rapid change). To assess the influence of these clinical and radiographic factors on the decisions of individual orthopaedic surgeons to operate (and to take into account factors such as waiting lists and differing criteria for operation for different surgeons) a separate study, specifically designed to assess these issues, is required.

There is no generally agreed method for subclassification of hip osteoarthritis or assessment of its severity. Several systems were therefore used in this large study. The Kellgren and Lawrence system for grading severity has been widely used and is certainly reproducible and a useful overall measure of severity. It does, however, lack sensitivity when assessment of change is required and, in particular, there is no mechanism for assessing further progression of change once severe osteoarthritis (Kellgren grade 4 ) is present. This was a particular problem in this study as over half the hips had severe changes at the time of presentation. In contrast the modification of Thomas's grading system is more time consuming and less reproducible ${ }^{23}$ but allows the detection of more subtle radiographic changes and hence may be more appropriate for prospective studies. In our experience, however, a global overall assessment of change by direct comparison of sequential radiographs is also of value.

The lack of progression in 53 and $46 \%$ of hips (global change and change in individual features respectively) is of interest and has been documented, though at lower frequencies (approximately one third of cases), in two previous hospital based studies. ${ }^{1726}$ This was more common in hips with mild radiographic osteoarthritis at presentation. The lack of radiographic progression does not necessarily associate with good symptomatic outcome, however, with $88 \%$ of patients reporting worsening of symptoms and $66 \%$ having increasing pain.

The differences in radiographic progression according to the pattern of femoral head migration are in keeping with previous studies $^{14} 1718$; a higher occurrence of global radiographic change and change in individual features occurred in hips with superior migration and a lower occurrence in those with medial and axial migration. The superolateral pattern in particular showed an increased frequency of change in all radiographic features and this may, in part, relate to the mechanical stresses imposed in this pattern. A higher frequency of increased pain was found in patients with superolateral migration. This has previously been reported ${ }^{14} 17$ and related to the degree of capsular thickening seen in this pattern. ${ }^{14}$

We also found an increased occurrence of radiographic progression, and in particular rapid progression, in hips with an atrophic bone response. This supports the proposed poor outcome for hips with a limited bone response and where retaining osteophytes are not formed. ${ }^{18}$ The worse prognosis for patients who are older at symptom onset may in part be explained by their increased occurrence of atrophic bone response. ${ }^{23}$ Case reports ${ }^{19-21}$ have identified hips showing rapid destructive change in association with chondrocalcinosis and, though no association was found between progression (rapid or slow) and chondrocalcinosis in this study, three cases of rapid progression seen in patients with chondrocalcinosis were identified.

The increased frequency of radiographic change (global and individual features) and worsening symptoms and disability in women confirms that they develop a more severe form of hip osteoarthritis. This has previously been suggested; indirectly, with men having symptoms for longer before a hip operation, ${ }^{15}$ and directly with women showing more pain, disability, and radiographic change. ${ }^{27}$ Other studies, however, have not shown such sex differences. ${ }^{17}$

Obesity, as measured by the body mass index, was not found to associate with progression, change in pain, or increased disability. The role of obesity in progression of osteoarthritis of the hip remains unclear, with some workers finding increased pain ${ }^{17}$ whereas others find no influence on symptoms or radiographic change. ${ }^{26}$ One report found a higher average weight among patients showing radiographic 'improvement' when compared with those showing progression. ${ }^{18}$ This same study also found an increase in the occurrence of Heberden's nodes in the 'recovery' group: we found no such significant association.

Non-steroidal anti-inflammatory drugs, particularly indomethacin, have also been incriminated in the rate of progression of osteoarthritis of the hip. ${ }^{28-30}$ Recruiting patients at the time of hospital referral, however, made previous non-steroidal antiinflammatory drug use impossible to analyse; prospective, comparative trials are required to address this issue. In addition, though this study is of relevance to hospital referred patients, a community based study, containing a broader spectrum of severity and asymptomatic hip osteoarthritis, might find different associations and predictors of clinical and radiographic progression.

This large study suggests that certain patient and radiographic characteristics at the time of hospital referral can identify patients with poor radiographic and symptomatic outcome from hip osteoarthritis. In particular, the migration and bone response patterns at the hip, female 
sex, and having late initial symptom onset are important.

JL is a Roussel (UK) osteoarthritis research fellow. We are additionally grateful to Trent Regional Health Authority for financial support, and Professor A Wallace, Mr I W Forster, and $\mathrm{Mr} \mathrm{C} \mathrm{J} \mathrm{Howell} \mathrm{for} \mathrm{assistance} \mathrm{in} \mathrm{patient} \mathrm{recruitment.}$

1 Resnick D, Niwayama G. Degenerative disease at extraspinal locations. In: Resnick D, Niwayama G, eds. Diagnosis of bone and joint disorders. Vol. 2. London: Saunders, 1981: 1322-35.

2 Resnick D. Patterns of migration of the femoral head in osteoarthritis of the hip. Roentgenographic-pathologic correlation and comparison with rheumatoid arthritis. $A m$ f Roentgenol 1975; 124: 62-74.

3 Gofton J P. Studies in osteoarthritis of the hip: part 1. Classification. Can Med Assoc f 1971; 104: 679-83.

4 Hayward I, Bjorkengren A G, Pathria M N, Zlatkin M B, Sartoris D J, Resnick D. Patterns of femoral head migration in osteoarthritis of the hip: a reappraisal with migration in osteoarthritis of the hip: a reappraisal with CT and pathologic correlation. Radiology 1988; 166:

5 Solomon L. Patterns of osteoarthritis of the hip. $\mathcal{F}$ Bone foint Surg [Br] 1976; 58: 176-83.

6 Marks J S, Stewart I M, Hardinge K. Primary osteoarthritis of the hip and Heberden's nodes. Ann Rheum Dis 1979; 38: $107-11$.

7 McGoldrick F, O'Brien T. Osteoarthritis of the hip and Heberden's nodes. Ann Rheum Dis 1989; 48: 53-5.

8 Meachim G, Whitehouse G H, Pedley R B, Nichol F E, Owen $R$. An investigation of radiological, clinical and pathological correlations in osteoarthritis of the hip. Clin Radiol 1980; 31: 565-74.

9 Jeffery A K. Osteophytes and the osteoarthritic femoral head. $\mathcal{F}$ Bone foint Surg [Br] 1975; 57: 314-24.

10 Resnick D. Osteophytes of the femoral head and neck. Arthritis Rheum 1983; 26: 908-13.

11 Solomon L. Geographical and anatomical patterns of osteoarthritis. Br F Rheumatol 1984; 23: 177-80.

12 Goften J P. A classification of osteoarthritis of the hip and its relevance to pathogenesis. $\mathcal{F}$ Rheumatol $1983 ; 10$ (suppl 9): 65-6.

13 Hermodsson I. Roentgen appearance of coxarthrosis. Relationship between the anatomy, pathologic changes, and roentgen appearance. Acta Orthop Scand 1970; 41: 169-87.

14 Cameron H U, Macnab I. Observations on osteoarthritis of the hip joint. Clin Orthop Rel Res 1975; 108: 31-40.

15 Macys J R, Bullough P G, Wilson P D. Coxarthrosis: a study of the natural history based on a correlation of clinical, radiographic, and pathologic findings. Semin Arthritis Rheum 1980; 10: 66-78.

16 Harrison M H M, Schajowicz F, Trueta J. Osteoarthritis of the hip: a study of the nature and evolution of the disease. F Bone foint Surg [Br] 1953; 35: 598-626.

17 Danielsson L G. Incidence and prognosis of coxarthrosis. Acta Orthop Scand 1964; 66: 1-114.

18 Perry G H, Smith M J G, Whiteside C G. Spontaneous recovery of the joint space in degenerative hip disease. Ann Rheum Dis 1972; 31: 440-8.

19 Menkes C J, Decraemere W, Postel M, Forest M. Chondrocalcinosis and rapid destruction of the hip. $\mathcal{f}$ Rheumatol 1985; 12: 130-3

20 Menkes C J, Simon F, Delrieu F, Forest M, Delbarre F. Destructive arthropathy in chondrocalcinosis articularis. Arthritis Rheum 1976; 19: 329-48.

21 Gerster J C, Vischer T L, Fallet G H. Destructive arthropathy in generalised osteoarthritis with articular arthropathy in generalised osteoarthritis with

22 Spector $T$. The fat of the joint: osteoarthritis and obesity. f Rheumatol 1990; 17: 283-4.

23 Ledingham J, Dawson S, Preston B, Milligan G, Doherty $M$. Radiographic patterns and associations of osteoarthritis of the hip. Ann Rheum Dis 1992; 51: $1111-6$.

24 Kellgren J H, Lawrence J S. Radiological assessment of osteoarthritis. Ann Rheum Dis 1957; 16: 494-502.

25 Thomas R H, Resnick D, Alazraki N P, Daniel D, Greenfield R. Compartmental evaluation of osteoarthritis of the knee. Radiology $1975 ; 116: 585-94$

26 Seifert M, Whiteside C G, Savage O. A 5 year follow-up of fifty cases of idiopathic osteoarthritis of the hip. Ann Rheum Dis 1969; 28: 325-6.

27 Jorring K. Osteoarthritis of the hip. Epidemiology and clinical role. Acta Orthop Scand 1980; 51: 523-30.

28 Ronningen $\mathrm{H}$, Langerland $\mathrm{N}$. Indomethacin treatment in osteoarthritis of the hip joint. Acta Orthop Scand 1979; 50: 169-74.

29 Newman N M, Ling R S M. Acetabular bone destruction related to non-steroidal anti-inflammatory drugs. Lancet 1958; ii: $11-4$

30 Rashad S, Revell P, Hemingway A, Low F, Rainsford K, Walker F. Effect of non-steroidal anti-inflammatory drugs on the course of osteoarthritis. Lancet 1989; ii: 519-22. 